\title{
Image Restoration based on A Hybrid Model by Alternating Direction Optimization
}

\author{
Weifeng Zhou ${ }^{123}$ \\ School of Mathematics and Physics, Qingdao University of Science and Technology \\ Qingdao, China 266071 \\ School of Computer Science and Technology, Shandong University \\ Jinan, China 250201 \\ E-mail: wfzhouqde163.com
}

\section{Guangwei Xu}

School of Mathematics and Physics, Qingdao University of Science and Technology

Qingdao, China 266071

E-mail:wuguangwei@mail.dlut.edu.cn

In order to better preserve the edge features and also refrain them from the staircasing effect in smooth regions, this paper investigates a hybrid model for restoring the blurred images with additive Gaussian noise and an effective iterative scheme based on alternating direction method is employed to solve the minimization problem suggested by us with two regularization terms. Numerical experiments demonstrate that the hybrid scheme proposed by us is superior to the model with only one regularization term in respect of image restoration quality.

CENet2015

12-13 September 2015

Shanghai, China

\section{${ }^{1}$ Speaker}

${ }^{2}$ Correspongding Author

${ }^{3}$ This work was supported by the grants from Natural Science Foundation of Shandong Province (ZR2015PF001), the Foundation of China Postdoctoral (2015M570594), the Department of Education Foundation of Yunnan Province (2014Y446) and the Talent Start-up Fund of Qingdao Science and Technology University (010022670). 


\section{Introduction}

The image denoising and deblurring plays a significant role in the field of image processing. Finding the unknown original image $u$ from an observed image $u_{0}$ can be modeled as follows :

$$
\min _{u}\left\|K u-u_{0}\right\|_{2}^{2}+\lambda \Phi(u)
$$

where $K$ is a bounded linear operator, $\Phi(u)$ denotes a regularization function to overcome the ill-posed property and the regularization parameter $\lambda>0$ is used to balance the two terms. In 1992, Rudin, Osher and Fatemi [1] firstly put forward to consider the following $l$-1norm of the gradient of $u$ as the regularization scale

$$
\min _{u}\left\|K u-u_{0}\right\|_{2}^{2}+\lambda\|D u\|_{1}
$$

This total variation (TV) restoration model allows for discontinuities and turns out to do better in suppressing noise and handling edges; however, although the TV-based model makes great progress in preserving edges while filtering out noise, the visually unpleasant staircasing effects still emerge in the result images, which can be usually viewed as false edges by some sensitive detection tools. In order to solve this problem, high order partial differential equation based on image restoration methods has been investigated [2-3], for instance, the following LLT smoothing scheme [4]

$$
\min _{u}\left\|K u-u_{0}\right\|_{2}^{2}+\lambda\left\|D^{2} u\right\|_{1}
$$

These higher order filters succeed in avoiding piecewise constant of the solution, but the edge blur phenomenon still appears. Consequently, some hybrid smoothing methods have been developed [5-6]. Particularly, the total generalized variation (TGV) and a tensor based high order regularization scheme demonstrated their superiority when compared with LLT model [7]. A method based on wavelet sparse operator and TV regularization was applied to Poisson noise removal [8]. Li et al. designed an adaptive hybrid denoising model but featured low efficiency [9]. Recently, a learning-based method was proposed to improve the processing quality but it was time-consuming, just like some other learning technologies [10].

Here, we take the following hybrid model into consideration

$$
\min _{u}\left\|K u-u_{0}\right\|_{2}^{2}+\alpha\|D u\|_{1}+\beta\left\|D^{2} u\right\|_{1}
$$

where $K$ denotes the blurring operator, gradient operator $\|D u\|_{1}=\sqrt{u_{x}^{2}+u_{y}^{2}}$ and $\left\|D^{2} u\right\|_{1}=\sqrt{u_{x x}^{2}+u_{x y}^{2}+u_{y x}^{2}+u_{y y}^{2}}$. This hybrid regularization strategy can take account of advantages of the two filter terms and cover their shortcomings so as to yield more desired images.

This paper is organized as follows. In Section 2, the numerical algorithm for solving the proposed strategy is introduced; the contrast of the proposed method and the TV-based one by numerical experiments is given in Section 3; finally, the main conclusion is made in Section 4.

\section{Iterative Algorithm}

Although the hybrid model compromises the merits of the model with only one regularization, the complexity increases the computational difficulties. We introduce the following alternating direction method (ADM) to solve this problem, which stands up to be competitive with the previous and existing algorithms such as TwIST [11], FISTA [12], FTVd[13], etc. 


$$
\begin{aligned}
u^{k+1} & =\arg \min _{u}\left\|K u-u_{0}\right\|_{2}^{2}+\frac{\lambda}{2}\left\|u-w^{k}+b_{1}{ }^{k}\right\|_{2}^{2}+\frac{\lambda}{2}\left\|u-z^{k}+b_{2}{ }^{k}\right\|_{2}^{2} \\
w^{k+1} & =\arg \min _{u} \alpha\|D w\|_{1}+\frac{\lambda}{2}\left\|u^{k+1}-w^{k}+b_{1}{ }^{k}\right\|_{2}^{2} \\
z^{k+1} & =\arg \min _{u} \beta\left\|D^{2} z\right\|_{1}+\frac{\lambda}{2}\left\|u^{k+1}-z+b_{2}{ }^{k}\right\|_{2}^{2} \\
b_{1}{ }^{k+1} & =b_{1}{ }^{k}+u^{k+1}-w^{k+1} \\
b_{2}{ }^{k+1} & =b_{2}{ }^{k}+u^{k+1}-w^{k+1}
\end{aligned}
$$

Clearly, $u$ subproblem can be solved exactly, i.e.,

$$
u^{k+1}=\left(2 K^{T} K+2 \lambda\right)^{-1}\left[2 K^{T} u_{0}+\lambda\left(w^{k}-b_{1}{ }^{k}\right)+\lambda\left(z^{k}-b_{2}{ }^{k}\right)\right]
$$

The Bregman method suffers from difficulty in dealing with high-order problems, we conformably adopt the Chambolle's dual method [14-15] to solve $w$ and $z$ subproblems.

Algorithm 1: Alternating direction method to solve the model

Choose: parameters $\alpha, \beta, \lambda$ and time step size $\Delta t$;

Initialization: $b_{1}{ }^{0}=0, b_{2}{ }^{0}=0, p^{0}=0, w^{0}=0, w^{0}=u_{0}, z^{0}=u_{0}$

While $\frac{\left\|u^{k+1}-u^{k}\right\|_{2}}{\left\|u^{k}\right\|_{2}}>$ tol

$$
\begin{aligned}
& u^{k+1}=\left(2 K^{T} K+2 \lambda\right)^{-1}\left[2 K^{T} u_{0}+\lambda\left(w^{k}-b_{1}{ }^{k}\right)+\lambda\left(z^{k}-b_{2}{ }^{k}\right)\right] \\
& w^{k+1}=u^{k+1}+b_{1}^{k}-\frac{\alpha}{\lambda} \operatorname{div}\left(p^{k+1}\right) \\
& p_{i, j}{ }^{k+1}=\frac{p_{i, j}{ }^{k}+\Delta t\left(\mathrm{D}\left(\operatorname{div} p^{k}-\frac{\lambda}{\alpha}\left(u^{k+1}+b_{1}^{k}\right)\right)\right)_{i, j}}{1+\Delta t\left|\mathrm{D}\left(\operatorname{div} p^{k}-\frac{\lambda}{\alpha}\left(u^{k+1}+b_{1}{ }^{k}\right)\right)_{i, j}\right|} \\
& z^{k+1}=u^{k+1}+b_{2}{ }^{k}-\frac{\beta}{\lambda} \operatorname{div}^{2}\left(q^{k+1}\right) \\
& q_{i, j}{ }^{k+1}=\frac{q_{i, j}{ }^{k}-\Delta t\left(D^{2}\left(\operatorname{div}^{2} \mathrm{q}^{k}-\frac{\lambda}{\beta}\left(u^{k+1}+b_{2}{ }^{k}\right)\right)\right)_{i, j}}{1+\Delta t\left|D^{2}\left(\operatorname{div}^{2} \mathrm{q}^{k}-\frac{\lambda}{\beta}\left(u^{k+1}+b_{2}{ }^{k}\right)\right)_{i, j}\right|} \\
& b_{1}^{k+1}=b_{1}^{k}+u^{k+1}-w^{k+1} \\
& b_{2}^{k+1}=b_{2}^{k}+u^{k+1}-w^{k+1}
\end{aligned}
$$




\section{Numerical Results}

The following signal noise ratio (SNR) is adopted,

$$
S N R=10 \log _{10} \frac{\|u-\bar{u}\|_{2}^{2}}{\|n-\bar{n}\|_{2}^{2}}
$$

where $u, \bar{u}$ and $\bar{n}$ stand for the recovered image, the means of $\bar{u}$ and the noise $\bar{n}$ respectively.

Fig. 1(a) and Fig. 1(b) show the original "lenna" and the degenerated "lenna" image with $\mathrm{SNR}=14.55$, corrupted by Gaussian noise and blurred by 33 Gaussian kernel and standard deviation; 2. Fig. 1(c-e) are recovered images by TV, LLT and our proposed method by 19 outer iterations respectively. The consumption time of our method is about $0.42 \mathrm{~s}$ as to an image of the size of $256 * 256$ under Matlab 2009 in our Laptop with 2.9HZ. In the implementation of the algorithm proposed by us, the parameters are set to be $\alpha=0.004, \beta=0.0005, \lambda=0.5$ so as to yield more desired results. Generally speaking, the parameter $\alpha$ is larger than $\beta$ to save from the over-smoothing and $\lambda$ can be set around 1. Actually, $\lambda$ is used to make $w$ and $z$ approximate $u$, which is not so sensitive to the restoration results because of adding the update variables $b_{1}$ and $b_{2}$ [16]. The convergence curves of the methods with four different parameters are displayed in Fig. 4. Data 1 denotes the convergence curve of our proposed method with $\alpha=0.004, \beta=0.0005, \lambda=0.5$. Data 2 is the convergence of the scheme with $\alpha=0.004, \beta=10^{-10}, \lambda=0.5$, which approximates the TV regularization. Data 3 denotes the convergence behavior of the scheme with parameters $\alpha=10^{-10}, \beta=0.002, \lambda=0.5$, which approximates the LLT image restoration scheme. Larger $\beta$ will results in over-smoothing not only in LLT model but also in our proposed hybrid model. The hybrid model can obtain more desired results.

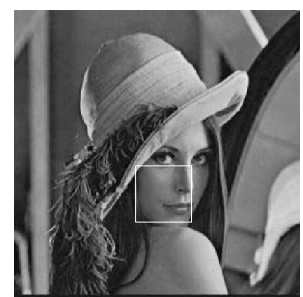

(a) Original Lenna

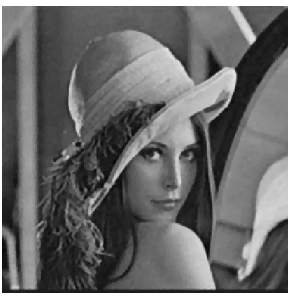

(c) TV

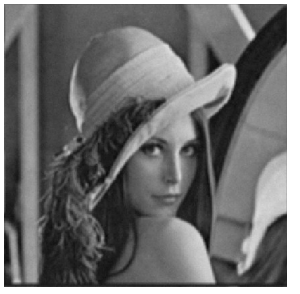

(d) LLT

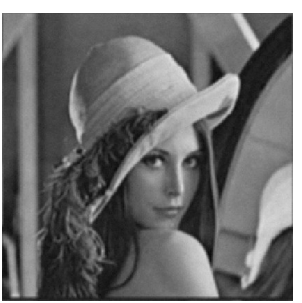

(b) Degenerated Lenna

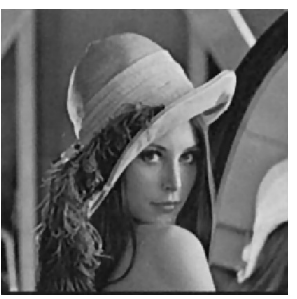

(e) hybrid result

Figure1: Results by TV-regularization, LLT Model and Our Scheme

\begin{tabular}{cclll}
\hline Lenna & Degenerated & TV & LLT & Our proposed \\
\hline SNR & 14.55 & 18.78 & 18.68 & 18.96 \\
\hline
\end{tabular}

Table 1: SNR Value of Observed Image and Recovered Images by Different Methods 
In comparison of the zoomed partial result image Fig. 2(c) processed by only TVregularization based algorithm with Fig. 2(d) processed by the algorithm proposed by us, we can clearly see that the disgusting staircasing effect emerges in the lenna's check in Fig. 2(c) and dramatically disappears in Fig. 2(d). Fig. 2(d) has more encouraging result and looks more natural. In comparison of the zoomed partial result image Fig. 3(c) processed by LLT model based algorithm with Fig. 3(d) processed by the algorithm proposed by us, we can find the edge preserving ability exceeds the LLT method. Our method has the advantages of both stair-casing reduction and edge-preserving accordingly.

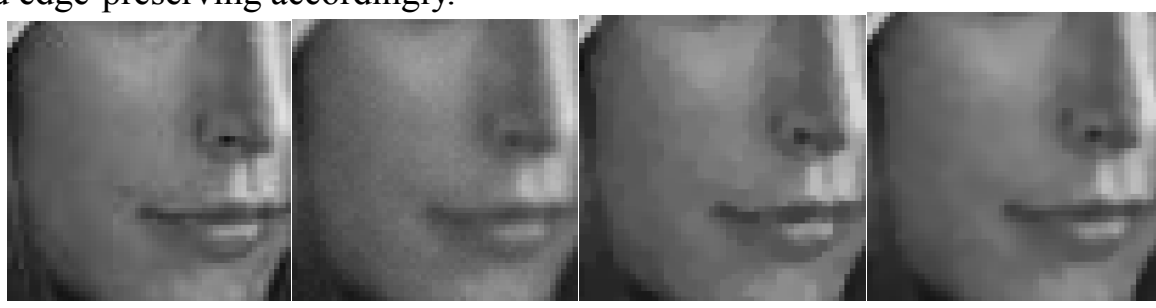
(a) Original Lenna
(b) Observed Lenna (c) TV
(d) Proposed Method

Figure 2: Zoomed Results by TV and Our Hybrid Scheme

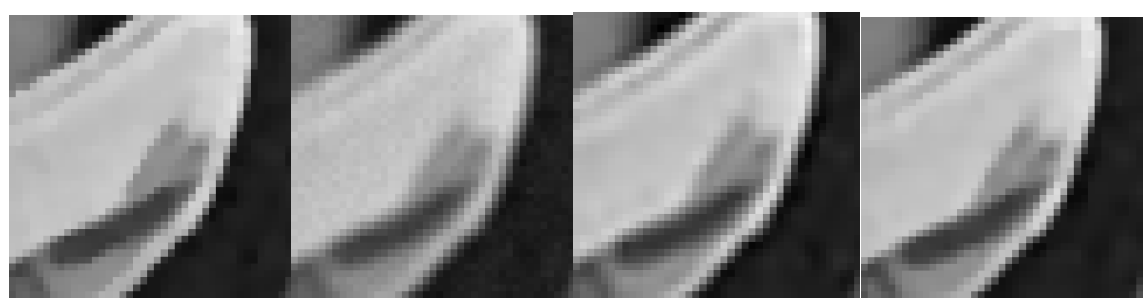
(a) Original Lenna
(b) Observed Lenna
(c) LLT
(d) Proposed Method

Figure 3: Zoomed Images by LLT Model and Our Scheme

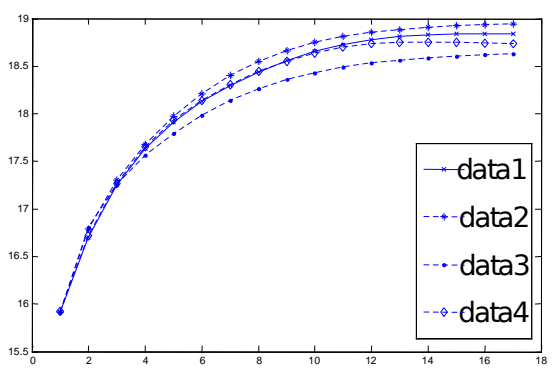

Figure 4: Convergence Curve

\section{Conclusion}

A hybrid regularization method for deblurring and denoising problem is introduced in this paper. In order to overcome the numerical calculation difficulties brought about by the nonsmooth high-order hybrid regularization term, the effective alternating direction method is hereby introduced. Simulation experiment results demonstrate that more high-quality reconstruction images are available by our proposed scheme. The images have not only preserved edges and but also avoids staircasing effects.

\section{References}


[1] L. Rudin, S. Osher, E. Fatemi, Nonlinear total variation based noise removal algorithms[J]Physica D 60: 259-268(1992)

[2] M. R. Hajiaboli,A self-governing fourth-order nonlinear diffusion filter for image noise removal[J]IPSJ Trans. Comput. Vision Appl. 2:94-103 (2010)

[3] Y. You, M. Kaveh, Fourth-order partial differential equations for noise removal[J]IEEE Trans. Image Process. 9 (10):1723-1730 (2000)

[4] M. Lysaker, A. Lundervold, X.-C. Tai, Noise removal using fourth-order partial differential equation with applications to medical magnetic resonance images in space and time[J]IEEE Tran. Image Process. 12 (12):1579-1590(2003)

[5] M. Lysaker, X.-C. Tai, Iterative restoration combining total variation minimization and a second-order functional[J]Int. J.Comput. Vis. 66 (1): 5-18(2006)

[6] F. Knoll, K. Bredies, T. Pock, R. Stollberger, Second order total generalized variation(TGV)for MRI[J], Magnet. Reson.Med. 65(2):480-491(2011).

[7] V. De and L. Lim, Tensor rank and the ill-posedness of the best low-rank approximation problem[J] SIAM J.Matrix Anal.App. 30(3),1084-1127(2008)

[8] D.-Q. Chen, L.-Z. Cheng, Deconvolving Poissonian image by a noval hybrid variational model[J], J. Vis. Commun. Image R. 22(7):643-652(2011).

[9] F. Li, C. Shen, J. Fan, C. Shen, Image restoration combining a total variational filter and a fourth-order filter[J]J. Vis. Commun. Image Represent, 18(4): 322-330 (2007)

[10]Y. Quan, H. Ji and Z. Shen, Data-Driven Multi-scale Non-Local Wavelet Frame Construction and Image Recovery[J],J.Sci.Comput., 63(2):307-329 (2014)

[11]J. Biouscas-Dias, M. Figueiredo, A new TwIst: Two step iterative shrinkage/thresholding algorithms for image restoration[J],IEEE Int. Conf. Imag Process.16(12):2992-3004 (2007)

[12]A.Beck and M.Teboulle, A fast iterative shrinkage-thresholding algorithm for linear inverse problems[J] SIAM J. Imag. Sci, 2(1): 183-202(2009)

[13] Y. Wang, J. Yang, W. Yin, Y. Zhang, A new alternating minimization algorithm for total variation image reconstruction[J]SIAM J. Imaging Sci. 1(3): 248-272 (2008)

[14]A. Chambolle, An algorithm for total variation minimization and applications[J] J.Math. Imaging Vis. $20: 89-97$ (2004)

[15]H.-Z. Chen, J.-P. Song and X.-C. Tai, A dual algorithm for minimization of the LLT model[J]Adv. Comput.Math.31:115-130(2009)

[16]T.Goldstein and S.Osher, The split Bregman algorithm for L1 regularized problems[J],SIAM .J. Imag. Sci.,2 323-43(2009) 